\title{
Minimizing Employee Injury by Changing the Behavior Through Behaviour Based Safety
}

\author{
Pradeep Roy. K. S ${ }^{1}$, Arun Karthik. V. $\mathbf{M}^{1}$, Dr. Muthukumar. $\mathrm{K}^{2}$ \\ ${ }^{1}$ PG Student, ${ }^{2}$ Professor \\ Department of Mechanical Engineering, \\ Bannari Amman Institute of Technology, Sathyamangalam, Tamil Nadu, India
}

\begin{abstract}
Since worker wounds can altogether affect the whole association, work environment security is a noteworthy worry for managers and representatives in all businesses. The expenses related with working environment wounds, including restorative and rehabilitative costs, lost profitability, individual damage claims, OSHA infringement, and the time required for mischance examination, give organizations the motivating force to distinguish approaches to diminish these wounds. Generally, most organization wellbeing programs set the important duty on administration to guarantee security in the working environment. An option and dynamic approach that is right now being utilized in industry is Behavior Based Safety. Conduct Based Safety alludes to security programs that utilization encouraging feedback to change worker conduct with a specific end goal to counteract business related wounds and sicknesses. By executing a Behavior Based Safety program, associations can straightforwardly include representatives in damage counteractive action and urge them to accept greater accountability for work environment security.
\end{abstract}

\section{INTRODUCTION}

Globalization is compelling organizations in the assembling business to recognize approaches to limit costs keeping in mind the end goal to stay focused. Decreasing work environment wounds can altogether diminish generally speaking hierarchical expenses, and along these lines enhancing the organization's aggressive position. Therefore, as future security directors and business pioneers, it's imperative for building and innovation projects to furnish their understudies with wellbeing administration preparing. To advance work environment wellbeing, an assortment of security programs with different outcomes are being utilized. With most conventional wellbeing programs, administration accepts the foremost accountability to guarantee security in the working environment. Additionally, numerous conventional wellbeing programs tend to center around what representatives are fouling up, rather than empowering them by strengthening positive work conduct. A dynamic and viable approach at present being utilized to advance work environment wellbeing is Behavior Based Safety. Conduct Based Safety programs utilize encouraging feedback to change representative conduct so as to counteract business related wounds and ailments. By actualizing a Behavior Based Safety program, associations can straightforwardly include representatives in damage avoidance and give them the motivating force to accept greater accountability for their wellbeing. Research has shown that businesses who give representatives and administrators a technique to practice their characteristic worry for wellbeing receive numerous benefits: less episodes and wounds, decreased expenses, and a more positive and gainful work environment where chiefs can center around change as opposed to policing workers. A Behavioral Safety process is the main technique demonstrated to enhance safe practices through a positive, worker driven, and consistently enhancing process. A conduct procedure likewise enables organizations to saddle the intensity of organization and individual qualities for wellbeing. This makes a culture where administrators and representatives work securely for the correct reasons as opposed to one where workers basically take after techniques to maintain a strategic distance from discipline (McSween, 2003). A conduct based way to deal with security enables laborers to be all the 
more effectively engaged with determining, characterizing and surveying right wellbeing hones. Practices ought to be characterized and dispassionately portrayed with the goal that it is clear to everybody included precisely what is required The prerequisites for its fruitful usage are administration duty and authority, bolster from administrators, and an open and confiding in culture.

\section{Encouraging feedback}

Altering representative conduct to avert work environment wounds can be accomplished through the use of Positive Reinforcement hypothesis. Utilizing uplifting feedback to advance safe work conduct depends on the accompanying premises fundamental human conduct (Skinner, 1953):

1. Conduct produces results.

2. The sort of result delivered by a given conduct will decide if that conduct will be rehashed or kept away from.

3. Positive results increment the likelihood of conduct being rehashed or learned, and negative outcomes increment the likelihood of conduct being maintained a strategic distance from. As a wellbeing administration procedure, associations can utilize uplifting feedback to furnish laborers with the motivating force to work securely.

Conduct Based Safety programs center around what the representative is doing well and oversee uplifting feedback to energize the learning of positive work conduct. Along these lines, uplifting feedback can lessen working environment wounds by straightforwardly including representatives in damage counteractive action and urge them to accept greater accountability for working environment security.

\section{SEGMENTS OF BEHAVIOR BASED SAFETY}

Working securely necessitates that representatives learn new conduct. Since learning is a progressive procedure, the persistent fortification of positive conduct is required until the point that the coveted conduct is accomplished. Associations utilizing Behavior Based Safety programs normally utilize the procedure known as "molding" as the favored strategy to adjust conduct. With forming, slow changes in safe working conduct are fortified or compensated while the worker is figuring out how to function securely (Reece and Brandt, 2005). Extra minutes, the worker figures out how to function securely and accepts the chief accountability for his/her conduct, bringing about less work environment wounds. In the meantime, Behavior Based Safety eases administration of the real obligation regarding guaranteeing security in the work environment. Concentrating on genuine safe conduct is proactive and enables other applicable issues to be distinguished and amended before a mischance can happen. This approach cultivates a shared, critical thinking group including administration and representatives to recognize basic arrangements of sheltered and perilous practices. Once the practices are distinguished, forming can be utilized to enable representatives to figure out how to function securely.

\section{The Steps for Shaping an Employees Behavior}

Identify the particular change in conduct that is wanted, Identify business related explanations behind the worker to change his/her conduct, Get the laborer to consent to change his/her conduct ,Establish timetables to learn new conduct and screen the laborer's conduct, As the laborer's conduct enhances, regulate uplifting feedback and Continue to screen and strengthen the specialist's certain conduct

Diminishing mischances over the long haul requires duty, from workers as well as from administration (Geller, 2001).

Past execution should be assessed, and mischances and episode records explored, Ask questions that prompt clear objectives and long haul finish, Encourage suitable practices through prizes and Rewards must be attached to particular practices or results, and should be opportune and proper to the level of achievement.

A one size fits all approach isn't prescribed. Individuals and friends culture vary so the prizes and approach adjusted must be extraordinary. Prior to picking rewards, consider workers age, geographic area, racial and ethnic decent variety. Spigener of Behavioral Science Technology Inc. recognized the accompanying as basic for the accomplishment of the program:

The monetary help, administration of administration, and representative association at all levels, Build up the segments, distinguish usage steps, assemble assets, and be set up to manage obstructions that emerge, Every individual included needs to comprehend their part and accountabilities, Give required preparing so workers know how to 
distinguish in danger practices and how to give criticism that will prompt more secure execution and Guarantee that all offices and divisions are engaged with the security program

A basic and frequently ignored segment is the individual representative. Specialists need to get tied up with the program. At the point when the administration group, without representative conference, forces a framework on the workforce, laborers are typically impervious to the new program. It is indispensable that the workforce takes an interest in the basic leadership forms, be educated as the program creates and on how the program will influence them, and what they can due before its execution.

\section{USAGE}

Before beginning a Behavioral Safety process, the organization first needs an unmistakable photo of the association's present wellbeing program. Recognize qualities and expand on those and deal with territories that need change. An appraisal will build up a fundamental plan for the program. Enroll agents from all territories of the organization and enroll however much help and association as could be expected. Make possession by guaranteeing there is wide hierarchical help through the inclusion of administration and workers.

\section{CONFINEMENTS OF BEHAVIOR BASED} SAFETY

Perceiving the advantages related with Behavior Based Safety, certain restrictions should be comprehended and tended to for the program to keep on being fruitful.

\section{The Major Limitations of Behavior Based Safety}

The requirement for consistent observing and support of safe work conduct, The need to create distinctive kinds of support for various representatives, Need to build up a time span for the nonstop organization of support, Difficult to execute if representatives don't work with similar individuals all the time, Difficult to change instilled demeanors and practices and Limited achievement if there is high turnover

\section{THE ABC'S OF BEHAVIOR BASED SAFETY}

First characterize the objective conduct since it must be discernible and recordable, Record the events of this conduct and once the objective conduct is recognized, intercessions can start to change the conduct

Mediations include adjusting the outcomes for the conduct. This is the ABC model of conduct change: Activator - Behavior - Consequence-Person. Activators incorporate wellbeing signs, preparing, and decides and approaches that immediate conduct and are just valuable when it is resolved that security issues are an after-effect of workers not recognizing what to do. These are helpful in beginning to learn new conduct, yet not in propping the conduct up (McSween, 2003). Outcomes ought to be an aftereffect of the conduct, regardless of whether as acclaim, train or criticism. Outcomes are just to be utilized when individuals comprehend what to do however don't do as such all the time as these are the sparks of conduct. Individuals will act in a way predictable with past encounters since this is the individual segment. Past practices that created positive results will be rehashed. Past practices that delivered negative results or no outcome won't probably be rehashed

In a conduct based process, positive and prompt outcomes are matched with the alluring conduct as a continuous spark for change. At the point when done effectively, practices can be recognized, input given, and a vehicle for change gave (Geller, 2001).

Organizations some of the time abandon the procedure since they don't see quick outcomes. Conduct Based Safety is certainly not a handy solution. It takes a long haul administration duty. It includes the control of perils by building strategies in procedures and gear, gathering criticism from representatives about hindrances, and the condition of the program. Additionally, the designation of satisfactory time and assets as required.

\section{IMPARTING SAFETY INFORMATION}

Powerful correspondence is the foundation of a sound authoritative security culture. Research has demonstrated that associations with better security societies and exhibitions have a tendency to have workers who consistently speak with each other in an open, deferential way. Safe tasks necessitate that representatives have all the data they have to work inside the security program. It is vital to test how well data is conveyed to the workers and how well they can apply it. Inquiries to survey security correspondences and preparing incorporate 
Are representatives performing as indicated by formal and casual interchanges programs? And Is there a formal and casual correspondences programs?

\section{ADVANTAGES OF BEHAVIOR BASED SAFETY}

Numerous dynamic organizations are as of now utilizing Behavior Based Safety programs with positive outcomes.

\section{The Major Benefits Resulting From Behavior Based Safety}

Reduction in workplace injuries, Reduction in medical related costs, including rehabilitative costs, Allowing the employee to assume the principal responsibility for safety, Focus on positive reinforcement, which tends to promote permanent learning Fewer work related injury lawsuits, Fewer company and governmental accident investigations and Increased productivity due to reduced injury related to absenteeism and illnesses

\section{CASES OF SUCCESSFUL BEHAVIOR BASED SAFETY PROGRAMS}

There are numerous cases of how producing organizations are at present utilizing Behavior Based Safety. The creators chose the accompanying cases of how this method is being utilized. At Los Alamos National Laboratory, Los Alamos, New Mexico, wanted safe practices were energized utilizing positive strengthening results. Amid execution, the workforce made their own security activities to make the procedure worker driven. Laborers watched each other for around 10 to 15 minutes and gave criticism recognizing safe and in danger practices.

The means included:

Observing laborers, and distinguishing safe and in danger practices, providing input to the specialists and permitting criticism, compiling the information for examination and critical thinking.

Some portion of their prosperity was credited to the way that specialists were not made to feel that they were the issue yet rather, that they were the arrangement. They inferred that laborers normally perform in danger practices since obstructions to safe work regularly constrain specialists in clashing ways. A few cases included: Lack of satisfactory abilities or learning to perceive risks and react fittingly Employees discovered routes around wasteful authoritative frameworks, The nonattendance of prizes for following safe strategies.

Lack of responsibility for following risky practices, Poor outline of workstation, Lack of support or inaccessible apparatuses and hardware No accord on standard work methods, Personal elements influencing the worker, for example, weariness, solution, stress, or ailment, Past built up rehearses and Worker purposely opens themselves to dangers.

A critical part of the program was the input procedure. Input is basic since it gives a strategy to danger acknowledgment and announcing that stayed unknown. For the date-book year 2001 the greater part of the classifications consolidated demonstrated an expansion in the sheltered practices from $90 \%$ to 95\% of every four months. This included diseases, redundant injury, strains, gashes, punctures and injuries.

\section{EVALUATION}

Different appraisal instruments have been utilized to assess the achievement of Behavior Based Safety programs. Customary security frameworks normally center around following damage related episodes

These are results of conduct and to be fruitful in averting wounds it is critical to center around the potential for a mishap. This will enable the organization to make a move before somebody gets injured. Utilizing data about past episodes, organizations can be proactive as far as progressing practices and perceive how regularly representatives are getting along the safe versus in danger practices. For instance, one organization distinguished through damage records that they had an issue with consumes amid the midyear months. The organization realized that with the best possible utilization of gloves these wounds ought to be avoided. They utilized their conduct based security procedure to quantify how regularly workers were utilizing hot consuming gloves in circumstances where it was justified. They confirmed that levels of utilization of gloves were lower than anticipated, yet just under specific conditions. With this new data, they made the modifications important to build the event of the protected conduct by guaranteeing that representatives knew when they ought to utilize hot consuming gloves, making the gloves more accessible (the essential wellspring of the issue), and perceiving the proper utilization of hot consuming gloves with 
positive criticism when it was watched. The outcome was an abatement in consumes. By finding a way to actualize a wellbeing framework that enabled them to quantify conduct and include workers during the time spent perception and input, the organization could spare a large number of dollars and decrease the event of consume wounds.

This security execution record is considered among the plain best in the business. In a surveying their Behavior Based

Security program they thought that it was critical to characterize and measure wellbeing regarding recognizable, quantifiable practices. For instance, they:

Defined and classified every one of the components that ought to be available in a far reaching wellbeing program ,Created questions that test for the nearness or nonattendance everything being equal, including managerial structure furthermore, reviews, security program segments, correspondences, preparing and consistence, Assigned qualities to the relative significance of every component in light of its potential effect on staff, activities, money related effect and administrative consistence ,Recorded lacks and change openings, Created remedial plans and spending plans which organized exercises in view of thing esteem, Measured the activities and results, not the exercises.

The main objective of the administration group was to guarantee that these components were obviously characterized in methods and determinations. To test the sufficiency of the wellbeing administration structure and evaluate their present program they made inquiries:

Are present systems sufficient?, Are there building wellbeing audits and methodology?, Is there an arranging and audit structure for continuous security exercises? , Is there a record-keeping framework used to track segments of the program?

Koch attempted to create world-class wellbeing execution. It has received a program called Total Safety Culture that focuses on halting hazardous practices previously an occurrence happens

Close miss reports including at-home/driving occurrences - Safety can't be turned on and off since it needs to be instilled into every individual's way of life.

Pre-work security gatherings - Identify the potential wellbeing and natural perils and talks about how to deal with those risks .Conduct perceptions - Every representative is engaged to close down any action esteemed hazardous, Root-source examinations Dissects the episode by soliciting an arrangement from "Why?" questions. Utilizing different methodologies customized for their condition, associations, for example, Hewlett-Packard, Exxon Mobile Chemical, Pfizer Pharmaceuticals, L.L. Bean, and Johnson and Johnson have effectively executed Behavior Based Safety at their organizations.

\section{WHY FOCUS ON BEHAVIOR BASED SAFETY}

Representatives at times neglect to adjust to wellbeing systems notwithstanding when they have the fundamental learning and apparatuses, in light of different elements impacting them. Individuals are more mindful of those elements that can possibly cause hurt in light of the fact that conduct wellbeing approaches recognize and center around these negative factors ._An mischance is the final product of a grouping, generally activated by a hazardous conduct.

At the point when programs are estimated by occurrence and mischance rates to gauge execution, they have a tendency to be responsive as opposed to being proactive.

Security administrators need to proceed:

Observations of representative activities, Equipment checks, office stroll through agendas and Housekeeping

\section{CONCLUSION}

As future security chiefs and business pioneers, it's vital that designing and building innovation programs give their understudies have a comprehension of wellbeing and wellbeing administration. Since worker wounds can essentially affect the whole association, lessening work environment wounds is a noteworthy worry for organizations in the assembling business. Conduct Based Safety programs are at present being utilized as a compelling contrasting option to customary organization wellbeing programs. 
While customary wellbeing programs put the main duty regarding work environment security on administration, Behavior Based Safety programs utilize encouraging feedback to avert business related wounds and disease. In the meantime, Behavior Based Safety program straightforwardly include representatives in damage aversion by urging them to accept greater accountability for work environment wellbeing.

The important strides for actualizing Behavior Based Safety include: administration bolster, worker trust, money related duty and time. It must be recollected that Behavior Based Safety is certainly not a handy solution and there will be impediments that should be survived. The nonappearance of moment results can be disappointing. Additionally, there will be times when representatives don't take after the principles. In these circumstances the best activity is to take a seat with the worker and revive and re-underscore the organization rationality, tenets, approaches and methodology

\section{REFERENCES}

1. Geller, E. Scott. "Behavior-based safety in industry: Realizing the large-scale potential of psychology to promote human welfare." Applied and Preventive Psychology 10.2 (2001): 87-105.

2. Geller, S. E. (2001) "Keys to Behavior-Based Safety", Government Institutes.

3. Krause, R., Thomas et al. (1997). The Behaviorbased Safety Process: Managing Involvement for an Injury-Free Culture, 2nd edition, John Wiley \& Sons., Somerset, New Jersey, U.S.A.

4. McSween, T. E. (2003). The Values-Based Safety Process: Improving Your Safety Culture with Behavior-Based Safety, 2nd edition, John Wiley \& Sons Inc., Somerset, New Jersey, U.S.A.

5. Skinner, B. F. (1953). Science and Human Behavior. New York: Macmillan.

6. Reece, B., and Brandt, R. (2005). Effective Human Relations, 9th edition, Houghton-Mufflin Company, Boston, U.S.A. 